\title{
DNA Deformation in Electric Fields: DNA Driven Past a Cylindrical Obstruction
}

\author{
Greg C. Randall and Patrick S. Doyle* \\ Department of Chemical Engineering, Massachusetts Institute of Technology, \\ Cambridge, Massachusetts 02139
}

Received September 17, 2004; Revised Manuscript Received January 4, 2005

\begin{abstract}
We present a kinematic analysis and experimental study of DNA deformation in electric field gradients. Specifically, we investigate deformation near a large insulating cylinder with single molecule fluorescence videomicroscopy. Because the electrophoretic velocity field is a potential field, a kinematic analysis shows that local deformation of DNA in any electric field gradient is pure elongation, quantified by a strain rate and orthogonal axes of extension and compression. From the kinematics, we construct the electrophoretic Deborah number relating the competing effects of deformation in the field and the polymer elasticity. We report highly configuration-sensitive stretching at the front of the obstacle and affine compression in the region near the back stagnation point. Furthermore, the DNA also can extend both "pre-impact" and "post-impact" in this inhomogeneous extensional field. We find that field gradient induced deformation offers a simple way to extend and quickly compress DNA near surfaces in microdevices.
\end{abstract}

\section{Introduction}

There is a large incentive to both fundamentally understand methods of DNA deformation and to devise ways to stretch and compress DNA in microdevices at will. For example, studying DNA deformation is crucial to understand biomolecular processes that require conformation flexibility, such as the transport of viral DNA into cells. ${ }^{1}$ Furthermore, stretching and compression of DNA can be useful unit operations for lab-onchip devices; e.g., researchers are currently trying to scan and sequence a single fully stretched DNA strand

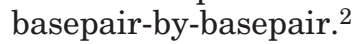

Flexible polymers like DNA deform from their native coil configurations in a solvent by applying forces either mechanically, with a hydrodynamic flow, or because DNA is negatively charged, with an electric field. For example, researchers have stretched tethered DNA with optical tweezers, ${ }^{3}$ uniform flows, ${ }^{4}$ and uniform electric fields. ${ }^{5}$ From the perspective of engineering continuous processes, it can be more interesting to study how free DNA deforms in a force field gradient. For example, in a hydrodynamic flow, any obstacle or channel contraction/expansion induces velocity gradients that deform a flexible polymer. Single polymer deformation in hydrodynamic flows (like uniform extensional flow, ${ }^{6,7}$ shear, ${ }^{8}$ or mixed flow ${ }^{9,10}$ ) has previously been observed and characterized. Similarly, electric field gradients created by obstacles or by a channel contraction/expansion presumably will deform DNA. There have been many studies of DNA electrophoresis through barriers and obstacles in microchannels ${ }^{11-18}$ where electrophoretic DNA stretching and compression may play an important but generally overlooked role. For example, our group has shown that the performance of a hooking hairpin DNA separation mechanism is highly dependent on electrophoretic stretching from obstacle induced gradients. ${ }^{17,18}$ In addition, simulation work has shown

* Corresponding author. MIT, Room 66-456, 77 Massachusetts Ave., Cambridge, MA 02139. Telephone: (617) 253-4534. Fax: (617) 258-5042. E-mail: pdoyle@mit.edu.

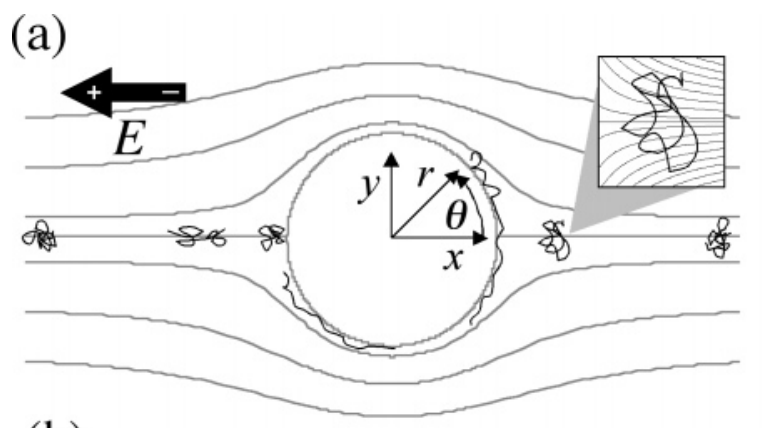

(b)

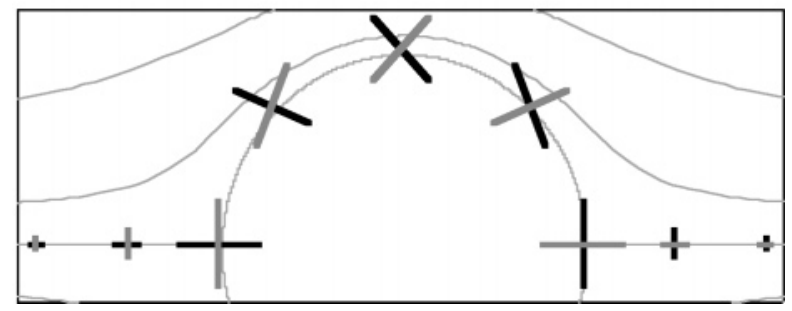

Figure 1. (a) Schematic of a DNA molecule electrically driven into an insulating obstacle (moving right to left). (b) Axes of extension (black) and compression (gray) along the centerline trajectory with magnitude scaled by the local electrophoretic strain rate $\dot{\epsilon}^{\mathrm{EL}}(r)$.

that DNA can deform electrophoretically in entropic traps, i.e., nanosized channel contractions, if the realistic curved electric field lines are incorporated. ${ }^{19}$

This recent work suggests that DNA deform from electric field gradients created in microfluidic devices, however no studies have addressed the details of how these charged polymers electrophoretically deform. In this paper, we focus on the dynamics of DNA deformation in electric field gradients, developing a kinematic model with experimental verification. As a case study, we choose the problem of electrophoretic deformation induced by a large nonconducting, cylindrical obstacle (Figure 1a). 


\section{Theoretical Background}

We consider large double-stranded DNA fragments, typically of size $50-1000 \mathrm{kbp}(\sim 0.01-1 \%$ of a typical human chromosome), and free of all in vivo proteins. The DNA molecule is primarily characterized by its persistence length $l_{\mathrm{p}}$, contour length $L$, diffusivity $D$, and longest relaxation time $\tau$. The large DNA considered here have $L \gg l_{\mathrm{p}}$ so that they adopt coiled configurations (with radius of gyration $R_{\mathrm{g}}$ ) at equilibrium in aqueous solvents. Backbone phosphate groups render DNA a uniformly negatively charged polymer at moderate $\mathrm{pH}$. In the presence of a uniform electric field $\mathbf{E}$, DNA coils move through solution at a size-independent velocity $\mu \mathbf{E}$ where $\mu$ is the electrophoretic mobility. ${ }^{20}$ Just as velocity gradients deform polymers in hydrodynamic flows, electric field gradients can deform polyelectrolytes during electrophoresis. Before we introduce electrophoretic deformation, we first review some basic aspects of hydrodynamic deformation that can be found in polymer fluids textbooks. ${ }^{21}$

Polymer deformation in hydrodynamic flows has been classified by kinematic models of the gradient in the flow field $\nabla \mathbf{u}^{\infty}$. We gain some physical insight by decomposing the velocity gradient tensor into its symmetric and antisymmetric parts, e.g. $\nabla \mathbf{u}^{\infty}=\boldsymbol{\Gamma}+\boldsymbol{\Omega}$, where $\boldsymbol{\Gamma}$ is the symmetric rate-of-strain tensor and $\boldsymbol{\Omega}$ is the antisymmetric vorticity tensor. It can be shown that $\boldsymbol{\Gamma}$ solely governs the local deformation of fluid elements whereas $\boldsymbol{\Omega}$ controls the local rotation of fluid elements. ${ }^{22}$ For comparison with electrophoretic stretching, we are primarily concerned with strong deformation and specifically, 2-dimensional (2D) extensional flows with $\boldsymbol{\Omega}=$ 0 . The local deformation in $2 \mathrm{D}$ extensional flows can be represented by a $2 \times 2$ symmetric matrix with (recalling the properties of symmetric matrices) eigenvalues $\pm \dot{\epsilon}$ and orthogonal eigenvectors $\mathbf{p}_{+}$and $\mathbf{p}_{-}$. When introduced to a $2 \mathrm{D}$ extensional flow field, an ideal flexible object will exponentially extend along the axis of extension $\mathbf{p}_{+}$and exponentially compress along the axis of compression $\mathbf{p}_{-}$at a rate governed by the strain rate $\dot{\epsilon}$. This ideal deformation is known as "affine deformation". Hence, deformation of polymers is analyzed with extension-strain plots where strain $\epsilon$ is the accumulated strain rate over a polymer's trajectory $\mathbf{x}(t), \epsilon=\int \dot{\epsilon}[\mathbf{x}(t)]$ $\mathrm{d} t$. Most previous work has been for homogeneous extensional flows so that $\epsilon=\dot{\epsilon} t_{\text {res }}$ where $t_{\text {res }}$ is the residence time in the flow. An affinely deforming object with initial size $x_{\mathrm{i}}$ will stretch to a length $x_{\mathrm{i}} \mathrm{e}^{\epsilon}$ after experiencing a strain of $\epsilon$. A flexible polymer in a strong extensional flow may initially deform affinely, but it generally deforms nonideally because of elasticity and the complexities of a coil configuration. ${ }^{6}$ A dimensionless parameter termed the Deborah number, defined as De $=\dot{\epsilon} \tau$, governs the competition between the diverging fluid elements dragging pieces of the polymer apart and the polymer's elasticity tending to recoil the chain. At a critical Deborah number of De $=1 / 2$, these competing effects exactly cancel and steady-state polymer configurations transition from coiled ( $\mathrm{De}<1 / 2$ ) to stretched (De $>1 / 2) .{ }^{23}$ Both experiments ${ }^{6}$ and simulations ${ }^{24}$ have confirmed that the dynamic deformation of single polymers in extensional flows with De $>1 / 2$ is highly sensitive to the polymer's initial configuration.

To examine how a charged polymer like DNA deforms in electric field gradients we must determine the dominant electric and hydrodynamic forces during electrophoretic stretching. Long et al. ${ }^{25}$ have previously addressed the simultaneous action of electric and hydrodynamic forces on polyelectrolytes. In their model, they discretize the polyelectrolyte as a bead-spring chain and linearly superimpose electric, hydrodynamic, and Brownian forces so that the velocity of one bead of the bead-spring model is

$$
\frac{\mathrm{d} \boldsymbol{x}_{n}}{\mathrm{~d} t}=\frac{1}{\xi}\left[\mathbf{T}\left(\mathbf{x}_{n}\right)+\mathbf{g}\left(\mathbf{x}_{n}\right)\right]+\mathbf{u}^{\infty}\left(\mathbf{x}_{n}\right)+\mu \mathbf{E}\left(\mathbf{x}_{n}\right)+\mathbf{u}_{\mathrm{HI}}\left(\mathbf{x}_{n}\right)
$$

where $\xi$ is the bead drag coefficient, and for the $n$th bead, $\mathbf{x}_{n}$ is the position vector, $\mathbf{T}\left(\mathbf{x}_{n}\right)$ is the force due to gradients in tension along the polymer, $\mathbf{g}\left(\mathbf{x}_{n}\right)$ is the Brownian force, $\mathbf{u}^{\infty}\left(\mathbf{x}_{n}\right)$ is the imposed flow field, $\mathbf{E}\left(\mathbf{x}_{n}\right)$ is the imposed electric field, and $\mathbf{u}_{\mathrm{HI}}\left(\mathbf{x}_{n}\right)$ is the induced velocity from hydrodynamic interactions from all other beads of the polyelectrolyte. Note that the pure hydrodynamic deformation discussed above occurs when $\mathbf{E}=$ 0 and both $\mathbf{u}^{\infty}$ and $\nabla \mathbf{u}^{\infty}$ are nonzero. In our study we will address electrophoretic deformation with $\mathbf{u}^{\infty}=0$ and both $\mathbf{E}$ and $\nabla \mathbf{E}$ nonzero. A general form for $\mathbf{u}_{\mathrm{HI}}\left(\mathbf{x}_{n}\right)$ is: ${ }^{25}$

$$
\mathbf{u}_{\mathrm{HI}}\left(\mathbf{x}_{n}\right)=\sum_{m} \mathbf{H}_{n m}\left[\mathbf{T}\left(\mathbf{x}_{m}\right)+\mathbf{g}\left(\mathbf{x}_{m}\right)\right]+\sum_{m} \mathbf{H}_{n m}^{\mathrm{EL}}\left[q_{m} \mathbf{E}\left(\mathbf{x}_{m}\right)\right]
$$

where $q_{m}$ is the charge of the $m$ th bead, $\mathbf{H}_{n m}$ mediates flow disturbances due to nonelectric forces from bead $m$ on bead $n$ (which is the Oseen tensor in the far-field limit), and $\mathbf{H}_{n m}^{\mathrm{EL}}$ mediates similar flow disturbances due to electrophoresis of a charged bead. The flow disturbance for the electrophoresis of a point charge is ${ }^{26}$

$$
\mathbf{H}_{n m}^{\mathrm{EL}}=\left(\mathbf{I}+\frac{\mathbf{x}_{n} \mathbf{x}_{m}}{r_{n m}^{2}}\right) \frac{\mathrm{e}^{-\kappa r_{n m}}}{8 \pi \eta r_{n m}}
$$

where $\mathbf{I}$ is the identity tensor, $r_{n m}$ is the distance between beads $n$ and $m, \eta$ is the solvent viscosity, and $\kappa^{-1}$ is the solvent Debye length. Note the exponential decays on the length scale of the Debye length. Therefore, for very thin Debye layers $\left(\kappa^{-1} \ll l_{\mathrm{p}}\right.$, generally the case for DNA electrophoresis buffers), $\mathbf{H}_{n m}^{\mathrm{EL}} \sim 0$. Physically, the hydrodynamic interactions are screened by the mobile counterions in the Debye layer moving in the opposite direction to the polyelectrolyte. Consequently, we see what Long et al. term "electrohydrodynamic equivalence", i.e., the hydrodynamic interactions are mediated by the Oseen tensor if the tension is generated by either electric or hydrodynamic deformation. With $\mathbf{H}_{n m}^{\mathrm{EL}} \sim 0$, eq 1 is like the Langevin equation for the Zimm model, ${ }^{27}$ so the governing polyelectrolyte dynamic parameters $(D$ and $\tau)$ are also independent of whether tensions are generated by electric or hydrodynamic forces. Therefore, a chain relaxing in a uniform $\mathbf{E}$ is equivalent to that in a uniform $\mathbf{u}^{\infty}$. Recall that this model is based on a bead-spring discretization of the polyelectrolyte. A different approach has been pursued numerically to account for the anisotropy of the electrophoretic mobility of a slender body by discretizing the polyelectrolyte as freely rotating ellipsoids. ${ }^{28}$ This alternative detailed model treats $\xi$ and $\mu$ as tensors which have slightly different forms for slender bodies. Such a discretization implies hydrodynamic stretch is slightly longer than an equivalent electrophoretic stretch near full extension. However experimental results ${ }^{5}$ for 
tethered $\lambda$ - to $3 \lambda$-DNA suggest that "electrohydrodynamic equivalence" persists even near $75 \%$ extension. In summary, no matter how tensions are created in the chain, either due to $\nabla \mathbf{u}^{\infty}$ or $\nabla \mathbf{E}$, they generate long-range hydrodynamic interactions. To analyze deformation of the chain in an electric field gradient, we invoke electrohydrodynamic equivalence to interrogate $\mu \mathbf{E}$ in the same fashion as the velocity field in hydrodynamic flows.

Although electrohydrodynamic equivalence dictates that local deformation of DNA is nearly identical from both $\nabla \mathbf{u}^{\infty}$ or $\nabla \mathbf{E}$ effects, the fields $\mathbf{u}^{\infty}$ and $\mathbf{E}$ are quite different in identical geometries. Recall that the primary sources of both flow and field gradients in microchannels are obstacles and channel contractions. The main difference between hydrodynamic and electric fields generated in these devices is the no-slip boundary condition required at all surfaces in hydrodynamic flows. Consequently, near a wall or obstacle surface, a flow field is locally "shear-like" with both extensional and rotational components. On the other hand, in the absence of time dependent magnetic fields, electrophoretic deformation is always locally pure elongation because the electrophoretic velocity gradient is symmetric (without a local rotational component) for a potential field $(\nabla \times \mu \mathbf{E}=$ $0)$. Of secondary importance, an electric field is a harmonic field in which field disturbances from obstacles with radius $R_{\text {obs }}$ decay as $R_{\text {obs }}{ }^{2} / r^{2}$, whereas a lowReynolds number hydrodynamic flow induces a creeping (biharmonic) flow in which disturbances to the flow from obstacles decay more slowly as $R_{\mathrm{obs}} / r$.

\section{Theoretical Framework}

As a case study, we investigate DNA deformation in the electric field near a large $\left(R_{\text {obs }} \gg R_{\mathrm{g}}\right)$ insulating cylinder. This problem serves as a prime example for many lab-on-chip applications which often contain obstacles, and the same analysis can be used for other geometries that create electric field gradients, such as a contraction or an expansion. The electrophoretic velocity of a charged object with mobility $\mu$ around an insulating cylinder in an obstacle-centered coordinate system is

$$
\begin{aligned}
& \mu \mathbf{E}(r, \theta)= \\
& \quad \mu E \cos \theta\left(\frac{R_{\mathrm{obs}}^{2}}{r^{2}}-1\right) \mathbf{e}_{\mathbf{r}}+\mu E \sin \theta\left(\frac{R_{\mathrm{obs}}^{2}}{r^{2}}+1\right) \mathbf{e}_{\theta}
\end{aligned}
$$

To avoid confusion between the DNA migration direction and the standard electric field convention (opposite), we explicitly denote the location of the \pm terminals on our electric field arrows, which point in the direction of DNA motion (Figure 1a). In all cases, we consider molecules impacting the cylinder from the right along the centerline $(\theta=0)$.

We seek to study the deformation of DNA in this field in the limit $R_{\text {obs }} \gg R_{\mathrm{g}}$. The electrophoretic velocity field gradient of eq 4 (in polar coordinates) is

$$
\mu \nabla \mathbf{E}(r, \theta)=\left[\begin{array}{cc}
\frac{-2 \mu E \cos \theta R_{\mathrm{obs}}{ }^{2}}{r^{3}} & \frac{-2 \mu E \sin \theta R_{\mathrm{obs}}{ }^{2}}{r^{3}} \\
\frac{-2 \mu E \sin \theta R_{\mathrm{obs}}{ }^{2}}{r^{3}} & \frac{2 \mu E \cos \theta R_{\mathrm{obs}}{ }^{2}}{r^{3}}
\end{array}\right]
$$

We can extract the electrophoretic strain rate $\dot{\epsilon}^{\mathrm{EL}}(r)$ by determining the eigenvalues:

$$
\pm \dot{\epsilon}^{\mathrm{EL}}(r)= \pm \frac{2 \mu E R_{\mathrm{obs}}^{2}}{r^{3}}
$$

and the axes of extension and compression by determining the eigenvectors:

$$
\mathbf{p}_{ \pm}(\theta)=\frac{1}{\sqrt{2 \mp 2 \cos \theta}}\left((\cos \theta \mp 1) \mathbf{e}_{\mathrm{r}}+\sin \theta \mathbf{e}_{\theta}\right)
$$

Figure $1 \mathrm{~b}$ shows the axes of extension and compression (with magnitudes scaled by $\dot{\epsilon}^{\mathrm{EL}}(r)$ ) for a centerline impact from the right. Note that both the strain rate and axes of extension and compression induced by an insulating cylinder are spatially inhomogeneous. The maximum value of the radial-dependent strain rate is $2 \mu E / R_{\text {obs }}$ at the obstacle surface and it falls off steeply away from the obstacle $\left(\sim R_{\mathrm{obs}}{ }^{3} / r^{3}\right)$. Thus, the strongest DNA deformation will occur near the obstacle surface. The axis of extension begins in the $y$-direction for an approaching molecule, however it rotates around the obstacle, finally ending in the $x$-direction at the backside. Thus, we may imagine that a flexible polyelectrolyte will extend perpendicular to the field on the front side of the obstacle, compress on the backside, and then extend in the field direction as it leaves the obstacle. Recall that the Deborah number De $=\dot{\epsilon} \tau$ governs polymer deformation in flow gradients. Since $\dot{\epsilon}^{\mathrm{EL}}(r)$ is inhomogeneous, we define De based on the maximum strain rate so that $\mathrm{De}=2 \mu E \tau / R_{\text {obs }}$, which makes intuitive sense since $\dot{\epsilon}^{\mathrm{EL}}(r)$ falls off rapidly away from the obstacle. However, it is important to remember that deformation can occur away from the obstacle, either before or after impact. Thus, we also define the local $r$-dependent Deborah number $\mathrm{De}_{\text {local }}(r)=\dot{\epsilon}^{\mathrm{EL}}(r) \tau$.

The inhomogeneous nature of the field requires special attention. Other groups have studied polymers deforming in inhomogeneous flows (for example, see refs 29-33). In particular, Panwar and Kumar ${ }^{33}$ observed that deformation is lower than in homogeneous flows and it is highly sensitive to the polymer's Brownian trajectory. Szeri, Wiggins, and Leal ${ }^{30}$ have theoretically addressed deformation kinematics in 2D inhomogeneous fluid flows. They represent a deformable object's orientation and length by a state vector, and show that the deformation of the state vector depends on the object's history because its equations of motion are nonautonomous. Consequently the strong stretching condition is not necessarily (using our variables) $\mathrm{De}_{\text {local }}(r)>1 / 2$. More generally, in an inhomogeneous field this strong stretching condition depends on the history of deformation, i.e., the object's initial orientation and path interval. Our case study of deformation near an insulating cylinder is an "open" problem as opposed to a time periodic problem, which means that it is not obvious what time to choose to evaluate the strong stretching condition. ${ }^{30}$ However, the problem simplifies along the obstacle centerline. On the centerline, the equilibrium orientation $\mathbf{p}_{+}$is constant which means that $\operatorname{De}_{\text {local }}(r)>1 / 2$ again satisfies the strong stretching condition. Our analysis only pertains to these centerline collisions. However, we do remark that off-center collisions can stretch, but these DNA will experience lower strain and the adverse effects of an inhomogeneous field. To summarize, a DNA molecule approaching the cylinder along the centerline experiences a pseudo-homogeneous 


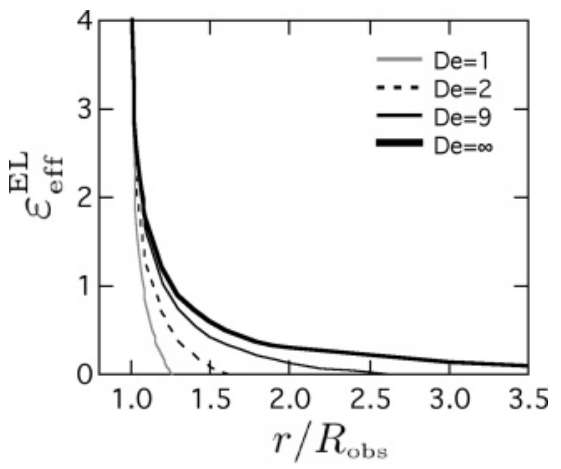

Figure 2. Effective integrated strain for a pointlike molecule approaching the obstacle stagnation point along the centerline $(\theta=0)$. This assumes no stretching occurs unless De $\operatorname{local}(r)>$ $1 / 2$. Note that $\epsilon^{\mathrm{EL}}=\lim _{\mathrm{De} \rightarrow \infty} \epsilon_{\mathrm{eff}}^{\mathrm{EL}}$.

extensional field, which we can envision as an extensional field quickly ramping to its maximal value at the obstacle surface with the local strain rate governing the strong stretching criterion.

We adopt a local analysis to address deformation away from the obstacle in the inhomogeneous field gradient. For this inhomogeneous field $\epsilon^{\mathrm{EL}}=\int_{0}^{\mathrm{t}_{\text {res }}} \dot{\epsilon}^{\mathrm{EL}}$ $[\mathbf{x}(t)] \mathrm{d} t$. We will present two options to compute electrophoretic strain for our study of centerline collisions: (1) assume $r=\infty$ at $t=0$ or 2) assume $r=r_{0}$ at $t=0$, where $r_{0}$ is the location where $\operatorname{De}_{\text {local }}(r)>1 / 2$. The first choice amounts to accumulating strain throughout the molecule's entire history whereas the second choice applies a De-dependent cutoff that initiates strain accumulation as soon as the electrophoretic strain rate is strong enough to induce a coil-stretch transition. We will term the accumulated strain as $\epsilon^{\mathrm{EL}}$ for the first option and $\epsilon_{\text {eff }}^{\mathrm{EL}}$ for the second. Using both definitions, we can quantify the pre-impact deformation on the front of the obstacle by computing the accumulated strain felt by a molecule before impact (neglecting diffusion). The accumulated molecular strain $\epsilon^{\mathrm{EL}}$ for centerline impacts $(\theta=0)$ approaching the front stagnation point is

$$
\epsilon^{\mathrm{EL}}(r)=\int_{\infty}^{r} \frac{\dot{\epsilon}^{\mathrm{EL}}(r) \mathrm{d} r}{\mu E(r)}=\ln \left[\frac{\left(r / R_{\mathrm{obs}}\right)^{2}}{\left(r / R_{\mathrm{obs}}\right)^{2}-1}\right]
$$

The same calculation can be performed by assuming that a molecule will not appreciably deform until $\mathrm{De}_{\text {local }^{-}}$ $\left(r_{\mathrm{o}}\right)>1 / 2$ :

$$
\epsilon_{\mathrm{eff}}^{\mathrm{EL}}(r)=\ln \left[\frac{\left(r / R_{\mathrm{obs}}\right)^{2}}{\left(r / R_{\mathrm{obs}}\right)^{2}-1} \frac{\left(r_{\mathrm{o}} / R_{\mathrm{obs}}\right)^{2}-1}{\left(r_{\mathrm{o}} / R_{\mathrm{obs}}\right)^{2}}\right]
$$

Note that $\epsilon^{\mathrm{EL}}=\epsilon_{\mathrm{eff}}^{\mathrm{EL}}$ in the limit De $\rightarrow \infty$ so that these two measures of strain only differ at low De. This is evident in Figure 2 which shows the expected accumulated strain $\epsilon_{\mathrm{eff}}^{\mathrm{EL}}$ as a function of De and distance from the obstacle. Note that molecules impacting the obstacle experience the same $\epsilon^{\mathrm{EL}}$ independent of De. However, Figure 2 shows that the amount of effective strain experienced in the strong part of the extensional field varies with De. As De decreases the effective strain before impact becomes more and more like a step function. For small De, an impacting molecule experiences a rapid transition from a negligible to a strong extensional field, whereas at higher De, pre-straining deforms the molecule as it approaches the obstacle. This is the important distinction between $\epsilon^{\mathrm{EL}}$ and $\epsilon_{\mathrm{eff}}^{\mathrm{EL}}$ and the primary reason we have defined $\epsilon_{\text {eff }}^{\mathrm{EL}}$. DNA deformation in the inhomogeneous field can be stronger at larger De due to both the larger electrophoretic strain rate and a larger $\epsilon_{\mathrm{eff}}^{\mathrm{EL}}$. Note that because of the $R_{\mathrm{obs}}{ }^{3} / r^{3}$ dependence of $\dot{\epsilon}^{\mathrm{EL}}$, the effective pre-impact strain is not large $\left(\epsilon^{\mathrm{EL}} \sim 1-1.5\right)$. However, the stretching of polymers in extensional flows is very sensitive to the initial configuration of the chain, ${ }^{6}$ so this pre-straining should not be dismissed.

With the above formalism, we seek to experimentally study DNA deformation in the electric field gradients induced by an insulating cylinder. Specifically, we will investigate single molecule extension-strain dynamics, the stretch-compress - stretch dynamics that arise from rotation of the axis of extension and compression, and the pre-strain effect.

\section{Experimental Section}

We used soft lithography ${ }^{34}$ to construct 5-10 mm-long, 100 $\mu \mathrm{m}$-wide, and $2 \mu \mathrm{m}$-high PDMS (poly(dimethylsiloxane)) microchannels with an isolated $10 \mu$ m-radius obstacle. A 10:1 PDMS: cross-linking agent (Sylgard 184, Dow) was degassed for $60 \mathrm{~min}$ at $15 \mathrm{in}$. of $\mathrm{Hg}$ vacuum pressure. The PDMS was then poured onto a Si master wafer with the negative of the microchannel geometry embedded in a layer of AZ 5214 image reversal photoresist (Clariant). The Si wafer was pretreated with a fluorinated silane monolayer (United Chemical Technologies) to prevent cured PDMS from sticking to the $\mathrm{Si}$ master. After pouring, the PDMS was allowed to degas for an additional hour at 15 in. of $\mathrm{Hg}$. The PDMS was then cured at $65{ }^{\circ} \mathrm{C}$ for $24 \mathrm{~h}$. Reservoirs $(4 \mathrm{~mm} \times 4 \mathrm{~mm})$ were cut at each end of the cured PDMS microchannel with a scalpel and the channels were soaked for $12 \mathrm{~h}$ at $50{ }^{\circ} \mathrm{C}$ in $0.5 \times$ TBE to eliminate permeation driven flow. ${ }^{35}$ Figure 3 a shows a SEM image of an isolated obstacle.

Monodisperse $\lambda$-DNA were stained with a fluorescent dye (TOTO-1, 4.7:1 bp:dye molecule) and diluted in one of two buffers: (1) $2.2 \times$ TBE, $3 \% \beta$-mercaptoethanol, $0.07 \% \operatorname{PVP}\left(M_{\mathrm{w}}\right.$ $=10^{6}, c^{*} \sim 1 \%$ ), and $0.07 \%$ ascorbic acid (viscosity $\eta=1.3 \mathrm{cP}$, $\mathrm{pH}=8.4$ ) or (2) $2.2 \times \mathrm{TBE}, 3 \% \beta$-mercaptoethanol, $0.07 \% \mathrm{PVP}$, $0.07 \%$ ascorbic acid, and $31 \%$ sucrose $(\eta=6 \mathrm{cP}, \mathrm{pH}=7.9)$. The viscosities were measured by bead tracking. ${ }^{36}$ We chose the high viscosity buffer to slow the dynamics to experimentally observable speeds. The additives were chosen to dynamically eliminate electroosmotic flow and scavenge oxygen. The measured electrophoretic mobility was $\mu=1.7 \pm 0.2(\mu \mathrm{m} / \mathrm{s}) /$ $(\mathrm{V} / \mathrm{cm})$ in the $1.3 \mathrm{cP}$ buffer and $\mu=0.25 \pm 0.03(\mu \mathrm{m} / \mathrm{s}) /(\mathrm{V} / \mathrm{cm})$ in the $6 \mathrm{cP}$ buffer. With the Debye approximation of the zeta potential, $\mu=\kappa^{-1} \sigma_{\mathrm{DNA}} /(4 \pi \eta)$ where $\sigma_{\mathrm{DNA}}$ is the DNA surface charge density. ${ }^{37}$ Thus, the observed decrease in mobility for the $6 \mathrm{cP}$ solvent is in the expected range given its higher viscosity $\left(\eta_{2} / \eta_{1} \sim 4.6\right)$, lower dielectric constant ${ }^{38}$ (so that $\kappa_{1}{ }^{-1}$ ) $\kappa_{2}{ }^{-1} \sim 1.1$ ), and lower $\mathrm{pH}$ (we calculate $\sigma_{\mathrm{DNA}, 1} / \sigma_{\mathrm{DNA}, 2} \sim 1.2 \mathrm{using}$ $\mathrm{p} K_{\mathrm{a}}$ values for $\mathrm{H}_{3} \mathrm{PO}_{4}$ along the DNA backbone). We acknowledge that this is a simplified check of how the mobility changes in different solvents and that other complex effects, specifically those from the dye interactions, may also be important to consider.

A typical experiment consisted of first gently rinsing and drying the microchannel and then applying it to a clean glass slide (plasma cleaned at $100 \mathrm{~W}$ for 5 min, charge equilibrated 1 day, soaked in $1 \mathrm{M} \mathrm{NaOH}$ for $15 \mathrm{~min}$ and rinsed in ultrapure water (Milli-Q, Millipore)). We then immediately filled the channel with DNA solution and applied an electric field across the reservoirs through platinum electrodes. We observed single DNA molecule dynamics using an inverted fluorescence microscope (Axiovert 200, Zeiss) with a $63 \times 1.4$ NA objective. Images were captured at 30 frames per second with an EBCCD camera (C7190-20, Hamamatsu) and NIH Image software. Digitized images had 8-bit pixel intensity values which ranged from 0 to 255 . 
(a)

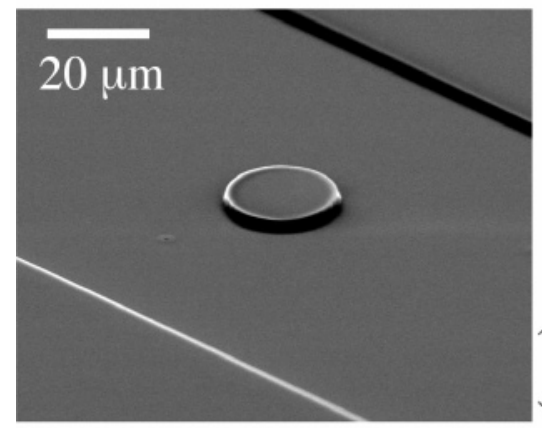

(b)

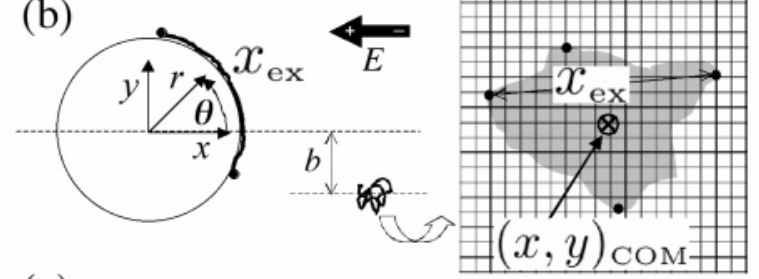

(c)

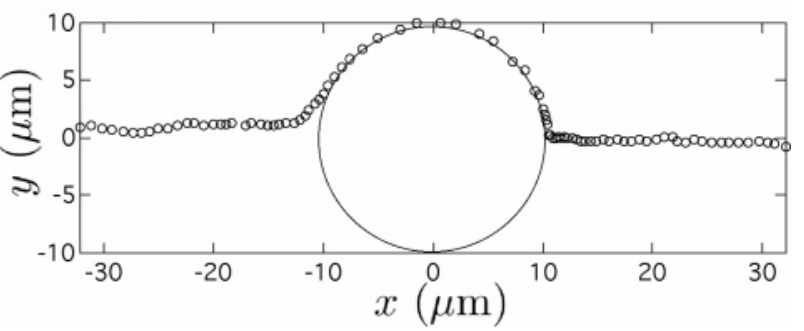

Figure 3. (a) SEM image of a $2 \mu \mathrm{m}$ high cylindrical PDMS post $\left(R_{\mathrm{obs}}=10 \mu \mathrm{m}\right)$. (b) Experimentally observable variables for an impact. (c) An example of a $\lambda$-DNA center of mass trajectory at $\mathrm{De}=2$ in the $1.3 \mathrm{cP}$ buffer. The time between each symbol is $1 / 30 \mathrm{~s}$.

Figure $3 \mathrm{~b}$ shows a cartoon of a typical digitized $\lambda$-DNA image from the $63 \times$ lens. At this magnification, the digitized image has a pixel size of $0.21 \mu \mathrm{m}$ square. For analysis, we filter the background by subtracting the maximum pixel value of the perimeter of the first frame of a movie from all the pixels in that movie. We extracted two observables from the captured images of a DNA molecule: the center of mass coordinate of the DNA $(x, y)_{\mathrm{COM}}$ and the DNA's longest extension $x_{\text {ex }}$. The center of mass of a molecule was computed by finding the first moment of the post-filtered image intensity distribution. ${ }^{39}$ This process was automated for multiple frames of data and it yielded the center-of-mass trajectory $\mathbf{x}(t)$ of the DNA (cf. Figure $3 c$ ). To determine the extension, we first located the coordinates of the left-most, right-most, top, and bottom pixels of the DNA image with an intensity value of at least 8 (after background filtering). The extension $x_{\text {ex }}$ is then defined as the length of the longest line connecting any two of these points (Figure 3b). For extreme extensions on the obstacle (i.e., if $x_{\text {ex }}$ $>7 \mu \mathrm{m}$ ), we manually locate the two end points of the chain and $x_{\mathrm{ex}}$ is the end-to-end arc length (Figure $3 \mathrm{~b}$ ). We define the impact parameter $b$ as the $y$-offset between the impacting DNA's center of mass and the obstacle center, taken at a distance $2 R_{\text {obs }}+R_{\mathrm{g}}$ from the obstacle center (Figure $3 \mathrm{~b}$ ). This position was chosen to evaluate $b$ because the DNA is far enough away from the obstacle to not experience high electric field gradients, yet close enough so that Brownian drift does not significantly coarsen our analysis. For this study, we only analyze centerline collisions with $|b|<1 \mu \mathrm{m}$. We can compute $\epsilon^{\mathrm{EL}}$ for a single DNA impact using its center-of-mass trajectory and the relation $\epsilon^{\mathrm{EL}}[\mathbf{x}(t)]=\sum_{\text {frames }} \epsilon^{\mathrm{EL}}\left(\mathbf{x}_{\text {frame }}\right) \Delta t_{\text {frame }}$ where the sum is over all frames in a movie, $\mathbf{x}_{\text {frame }}=(x, y)_{\text {COM }}$ is the center of mass in a given frame, and the frame rate $\Delta t_{\text {frame }}=1 / 30 \mathrm{~s}$. For different De, we begin data accumulation at a slightly different initial position $\mathbf{x}(0)$ based on limitations on our field of view and need for post-impact data. Therefore, we renormalize our $\epsilon^{\mathrm{EL}}$ data by adding in a small deterministic correction that is the molecular strain experienced before observation $\epsilon^{\mathrm{EL}}[\mathbf{x}(0)]$. Note that this correction is small $\left(\epsilon^{\mathrm{EL}}\right.$ $[\mathbf{x}(0)]<1 / 2)$. Therefore, our presented strain values are $\epsilon^{\mathrm{EL}}=$ $\epsilon^{\mathrm{EL}}[\mathbf{x}(t)]+\epsilon^{\mathrm{EL}}[\mathbf{x}(0)]$.

For analysis, we require $L$ and $\tau$ of stained $\lambda$-DNA. $L$ of unstained $\lambda$-DNA is $16.3 \mu \mathrm{m}$, however the intercalating TOTO-1 dye slightly unwinds the double helix and thereby increases its contour length. Using the results of Marko and Siggia ${ }^{40}$ the high-force extension of a tethered wormlike chain scales with the force $f$ as $f^{-0.5}$. By plotting the equilibrium extension vs $(\mu E)^{-0.5}$ for DNA over $50 \%$ extended hooked on small obstacles $\left(R_{\text {obs }}=0.8 \mu \mathrm{m}\right)$, we were able to extrapolate $L$ $=21 \mu \mathrm{m}$ for $(\mu E)^{-0.5} \rightarrow 0$ for this staining ratio. This agrees with other researchers who have found that using TOTO -1 increases a DNA's contour length anywhere from 4.1 to 4.8 $\AA$ Àdye molecule, which for us would correspond to $L=20.5-$ $21.3 \mu \mathrm{m} .{ }^{4,41,42}$ Furthermore, we used molecular combing ${ }^{43}$ to verify that the our fully stretched $\lambda$-DNA are on the order of $20-21 \mu \mathrm{m}$. The relaxation time $\tau$ for DNA has previously been determined to be $0.1 \eta \mathrm{s}$ in an aqueous buffer of viscosity $\eta$ with units of $\mathrm{cP} .{ }^{6}$ Specifically, this was done by examining chain relaxation in the linear force regime:

$$
\left\langle x_{\mathrm{ex}}(t) x_{\mathrm{ex}}(t)\right\rangle=\left(x_{\mathrm{i}}^{2}-\left\langle x^{2}{ }_{\mathrm{ex}}\right\rangle_{0}\right) \exp (-t / \tau)+\left\langle x^{2}{ }_{\mathrm{ex}}\right\rangle_{0}
$$

where $x_{\mathrm{i}}$ is the initial stretch (about 30\% extended for linear regime) and $\left\langle x^{2}{ }_{\text {ex }}\right\rangle_{0}$ corresponds to the mean square coil size at equilibrium. However, the confinement of our thin channels will increase $\tau .^{27,44}$ Therefore, we experimentally determined $\tau$ inside these $2 \mu \mathrm{m}$-high channels by obtaining $x_{\text {ex }}(t)$ data for stretched DNA (away from any obstacles or sidewalls). To stretch the DNA, we electrophoretically drive DNA into small obstacles ${ }^{17}$ to form symmetric hooked configurations. These DNA then unhook and advect in the field away from the obstacle in this strongly stretched state $\left(x_{\mathrm{ex}} / L>0.6\right)$. We track $x_{\mathrm{ex}}(t)$ for these relaxing DNA beginning when $x_{\mathrm{ex}}=x_{\mathrm{i}}=0.30$. We then fit the relaxation data to eq 10 with $\tau$ as the only free parameter. In these $2 \mu \mathrm{m}$-high channels, we obtain $\tau=$ 0.19 for the $1.3 \mathrm{cP}$ buffer and $\tau=0.9 \mathrm{~s}$ for the $6 \mathrm{cP}$ buffer. These increases in $\tau$ from confinement are consistent with simulations of DNA in confined channels. ${ }^{44}$

\section{Results and Discussion}

For DNA electrophoretically driven into a large insulating obstacle $\left(R_{\mathrm{obs}}=10 \mu \mathrm{m}\right)$ along its centerline $(|b|<1 \mu \mathrm{m})$, we investigate the pre-impact deformation, single molecule extension-strain dynamics, and the stretch-compress-stretch dynamics that arise from rotation of the axis of extension and compression. This kinematic analysis is based on deformable objects much smaller than the obstacle size. Using different size obstacles and field strengths, we have already confirmed and reported elsewhere ${ }^{17}$ that the PDMS obstacles are insulating and the observed critical coil-stretch transition occurs at $\mathrm{De} \sim 0.5$.

To quantify deformation it is first helpful to know how extended a DNA coil is in the absence of any electric field gradients. Diffraction of light will tend to smear and slightly enlarge our images of DNA coils. Figure 4 shows the experimentally measured probability distribution $P\left(x_{\mathrm{ex}} / L\right)$ of the equilibrium coil fractional exten- 


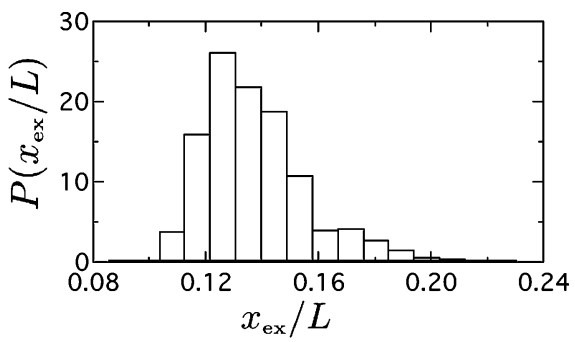

Figure 4. Equilibrium probability distribution of the DNA fractional extension.

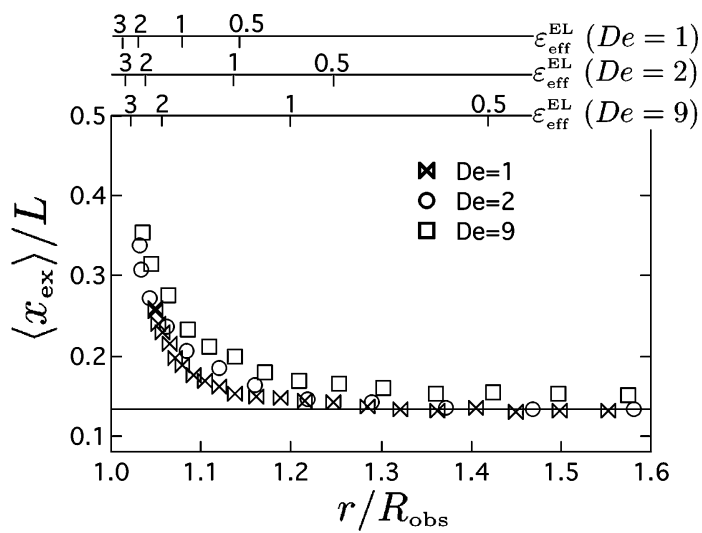

Figure 5. Average fractional extension of $\lambda$-DNA molecules approaching a large insulating cylinder (ensemble size $=30$ ). The upper axes give the effective accumulated strain and the horizontal baseline is the equilibrium average extension $\left(\left\langle x_{\mathrm{ex}}\right\rangle_{0} / L=0.13\right)$. The De $=9$ data is slowly approaching this baseline but our field of view did not allow observation beyond $r / R_{\text {obs }} \sim 1.6$.

sion for 1000 frames of images of $\lambda$-DNA using the 63 $\times$ lens. The mean of the distribution, denoted $\left\langle x_{\mathrm{ex}}\right\rangle_{0} / L$ is 0.13 . Because of blooming, ${ }^{45}$ this distribution is dependent on the camera, light source, lens, and dye ratio. Although we do not expect much more than a $10 \%$ departure from our measured $\left\langle x_{\mathrm{ex}}\right\rangle_{0} / L$ with large adjustments of the image capture settings, care was taken to keep all of these settings constant in all experiments.

In our examination of electrophoretic deformation, we first address the effect of the inhomogeneous electric field deforming the DNA molecules before impacting the large obstacle. The definition of "impact" is subtle since, in the absence of large normal forces, no DNA molecule is ever completely in direct contact with the obstacle because of a lubricating layer of solvent molecules. We empirically define "impact" with the obstacle when the leading edge of a DNA's fluorescent cloud has stopped moving toward the obstacle center. For $\lambda$-DNA in this study, impact typically occurs when the center of mass is at about $r / R_{\text {obs }} \sim 1.1$, although it can occur near $r / R_{\text {obs }}$ $\sim 1$ for molecules with a high degree of pre-impact deformation. Figure 5 shows the average fractional extension of DNA molecules approaching the obstacle along its centerline at $\mathrm{De}=1,2$, and 9 . As De increases, the degree of pre-impact deformation increases because of the combined effects of larger De and larger $\epsilon_{\text {eff }}^{\mathrm{EL}}$. However, as we presumed, this effect is small because these molecules only experience $\epsilon^{\mathrm{EL}} \sim 1$ before they impact the obstacle.

We now turn to single molecule extension-strain dynamics. Parts a-c of Figure 6 shows the fractional extension $x_{\mathrm{ex}} / L$ of single $\lambda$-DNA molecules colliding into the obstacle as a function of accumulated strain over each molecule's center of mass trajectory at $\mathrm{De}=1,2$,

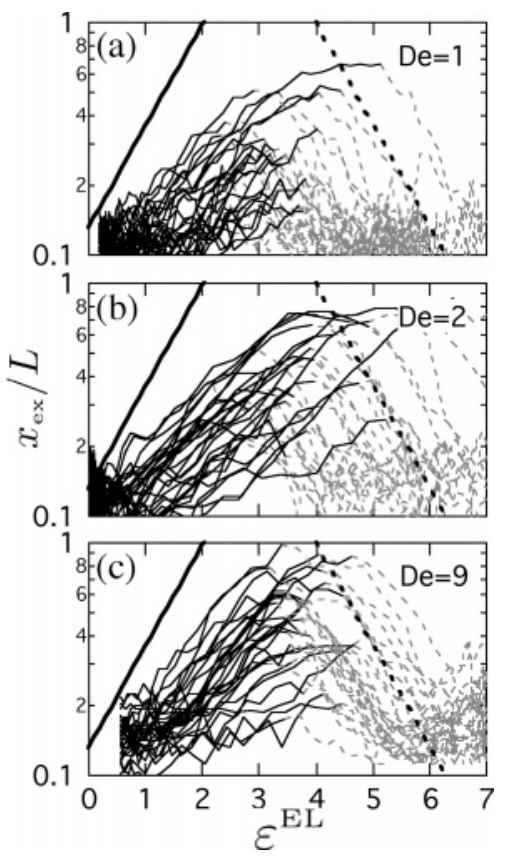

Figure 6. Extension-strain curves for $30 \lambda$-DNA molecules colliding into a large $\left(R_{\mathrm{obs}}=10 \mu \mathrm{m}\right)$ insulating cylinder at (a) $\mathrm{De}=1$, (b) $\mathrm{De}=2$, and (c) $\mathrm{De}=9$ along the centerline. Each curve represents the trajectory of a single molecule. Curves switch from solid black to dashed gray when a piece of the DNA crosses the plane $x=0$. Affine extension $\left(\sim \mathrm{e}^{\epsilon \mathrm{EL}}\right.$, solid line) and compression ( $\sim \mathrm{e}^{-\epsilon^{\mathrm{EL}}}$, dashed line) scalings are shown for reference.

and 9. Because of the small numerical difference between $\epsilon_{\text {eff }}^{\mathrm{EL}}$ and $\epsilon^{\mathrm{EL}}$ after impacting the obstacle, we chose to plot the data in terms of $\epsilon^{\mathrm{EL}}$. The transition from solid lines to dashed lines indicates that some portion of the molecule has crossed the bisecting plane $x=0$. By comparing the trajectories to the affine scaling $\left(\sim \mathrm{e}^{\mathrm{EL}}\right)$, we see an approach to affine deformation as De increases. We also note that the ensemble average fractional extension as a function of strain at $\mathrm{De}=2$ is in close agreement with the results from uniform extensional flow, ${ }^{7}$ a result not completely evident beforehand due to the inhomogeneity of this field and the electrophoretic deformation. This close similarity stems from electrohydrodynamic equivalence and the extremely fast decay of $\dot{\epsilon}^{\mathrm{EL}}(r)$ which gives a pseudohomogeneous extensional field. The diversity in extension-strain dynamics is reminiscent of hydrodynamic extensional flow studies, ${ }^{6,7}$ which show that the unraveling of an individual polymer is highly sensitive to its initial configuration. Perkins et al..$^{6,7}$ have previously observed that some polymer configurations (like a tight coil or a "folded" molecule) are more resistant to extensional deformation. Figure 7a, which shows an impact at $\mathrm{De}=2$, provides a visual example of a molecule that experiences significant strain $\left(\epsilon^{\mathrm{EL}}>3\right)$ near the front stagnation point, yet never appreciably stretches. For comparison, Figure $7 \mathrm{~b}$ shows a different molecule at the same De and comparable accumulated strain that significantly stretches.

Returning to Figure 6, parts a-c, note that the DNA begins to compress as it passes the $x=0$ plane. This was predicted by our previous kinematic arguments because the axes of extension and compression rotate along a given field line, and as a DNA molecule passes $x=0$, it becomes more closely aligned with the axis of compression. It is very interesting that almost every 
(a)

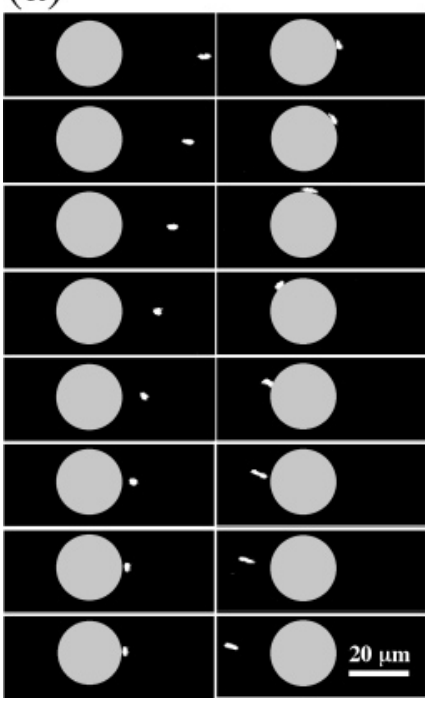

(b)

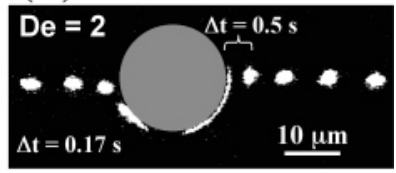

(c)

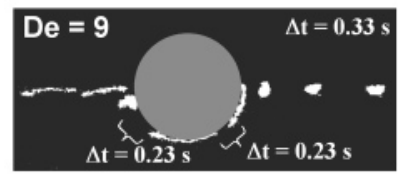

Figure 7. (a) Sample images of a $\lambda$-DNA collision at $\mathrm{De}=2$ (in $1.3 \mathrm{cP}$ buffer) without significant deformation. The time steps between frames are $0.1 \mathrm{~s}$. (b) A sample impact (rightto-left) at $\mathrm{De}=2$ in $1.3 \mathrm{cP}$ buffer with time steps of $0.17 \mathrm{~s}$ unless otherwise noted. (c) An impact at De $=9$ in the $6 \mathrm{cP}$ buffer with $0.33 \mathrm{~s}$ time steps unless otherwise noted.

(a)

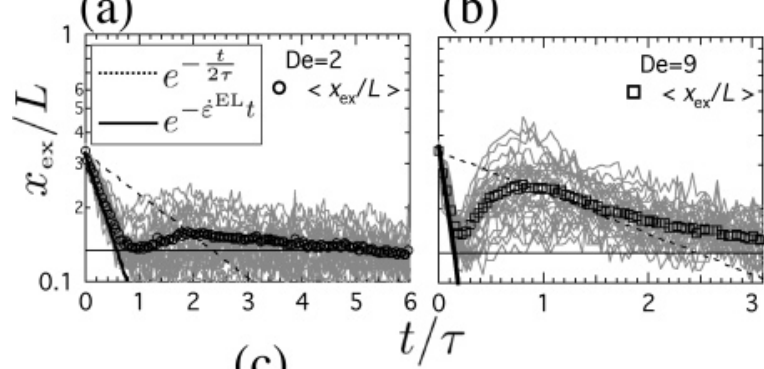

(c)

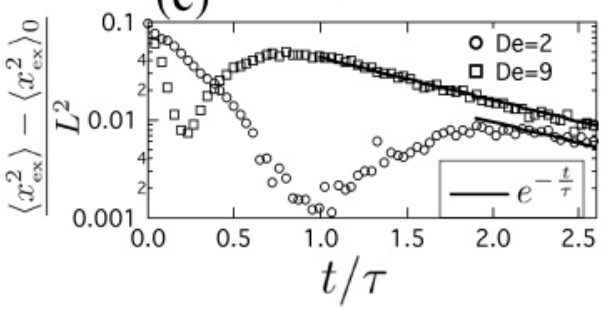

Figure 8. Dynamic extension during compression on the backside of a large obstacle at (a) $\mathrm{De}=2$ and (b) $\mathrm{De}=9$. Time was reset to zero when a compressing DNA had $x_{\mathrm{ex}} / L=0.3$. The thin horizontal line is $\left\langle x_{\mathrm{ex}}\right\rangle_{0} / L$. The gray lines are data for individual molecules (30 total) and the symbols are the ensemble average. The solid line scaling is the affine scaling and the dashed line scaling is the thermal relaxation scaling. (c) Mean square fractional extension with thermal relaxation scaling (solid lines) after the molecules leave the obstacle for the same data.

molecule compresses at the affine limit $\left(\sim \mathrm{e}^{-\epsilon^{\mathrm{EL}}}\right)$. Furthermore, note that some molecules stretch again after this compression. This trend grows stronger as De increases from 2 to 9 and can be easily visualized in Figure 7, parts b and c.

We now look more closely at the compression and post-impact stretch of DNA on the backside of the obstacle. Parts a and b of Figure 8 show $x_{\mathrm{ex}}$ vs $t / \tau$ of $\lambda$-DNA molecules deforming on the backside of the large obstacle at $\mathrm{De}=2$ and 9 . The time has been renormalized so that $t=0$ is when a stretched molecule has compressed to $x_{\text {ex }} / L=0.3$. We provide the equilibrium

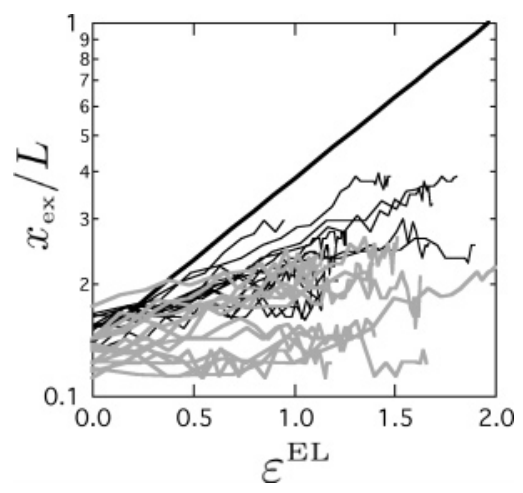

Figure 9. Dynamic post-impact extension at $\mathrm{De}=9$. The strain was reset to zero when the compressing DNA achieved a minimum $x_{\mathrm{ex}} / L$ near the back stagnation point. Black trajectories correspond to molecules $\geq 50 \%$ extended on the front of the obstacle, while gray trajectories are molecules which stretch $<50 \%$. Plotted trajectories terminate when the extension begins to drop due to relaxation. The solid line is the affine scaling.

fractional extension as a baseline and scalings for affine compression $\left(\left\langle x_{\mathrm{ex}}\right\rangle / L \sim \mathrm{e}^{-\dot{\epsilon}^{\mathrm{EL}} \mathrm{t}}\right)$ and thermal relaxation $\left(\left\langle x_{\mathrm{ex}}\right\rangle / L \sim \mathrm{e}^{-t /(2 \tau)}\right)$. Parts a and b of Figure 8 verify that the DNA molecules compressing at the obstacle backside recoil at the affine limit, faster than thermal relaxation. For example, at De $=9$, the DNA returns from stretched to coil configurations in $\sim 0.2 \tau$. Affine compression is expected because a stretched DNA on the backside is more closely aligned with the primary axis of compression, and when stretched out, there is little resistance to the compressive forces for such a flexible polymerthe DNA easily buckles and crumples into a compact configuration. Notice in parts $a$ and $b$ of Figure 8 that after ample compression on the obstacle backside, some molecules then extend along the axis of extension oriented in the $x$-direction (cf. Figure 7c). This effect is more prominent at high De, which we explain below by considering the details of the DNA configuration and the inhomogeneous strain rate.

To explain the stretching on the backside of the obstacle, or "post-impact stretch", it is helpful to highlight the differences between DNA molecules stretching at the front and at the back of the obstacle. We have already noted that the primary direction of post-impact stretch is in the $x$-direction whereas it is in the $y$ direction in the front. Deformation at the backside further differs from that at the front because the starting DNA conformation is not an equilibrium coil. For example, molecules which significantly stretch on the front of the obstacle are then compressed into "crumpled" configurations as they round the obstacle and do not have time return to an equilibrium configuration. Figure 9 shows the post-impact stretch data renormalized so that $\epsilon^{\mathrm{EL}}=0$ when the chain stretch $x_{\mathrm{ex}}$ takes its minimum value near the rear stagnation point. The population is divided into "crumpled" molecules that have strongly stretched on the front side $\left(x_{\mathrm{ex}} / L \geq 0.5\right)$ and those that only weakly stretched $\left(x_{\mathrm{ex}} / L<0.5\right)$. There is a strong correlation between molecules that were strongly stretched on the front and those molecules that extend faster at the backside. In general, we find that crumpled DNA configurations tend to stretch faster than equilibrium coils and speculate that this may be because they lack significant configuration complexities.

Continuing our comparison of stretching at the front and back of the obstacle, the local strain rate during 
backside stretching will also be weaker than at the front. This is because of the DNA's finite size ( $\lambda$-DNA has $\left.\left\langle x_{\mathrm{ex}}\right\rangle_{0}=2.8 \mu \mathrm{m}\right)$ and because the DNA is moving away from the obstacle as it stretches. For example, at a distance of $\left\langle x_{\mathrm{ex}}\right\rangle_{0}$ from the obstacle surface, De local $_{\text {lo }}$ is reduced to $1 / 2$ De. Consequently, a crumpled molecule leaving the back of an obstacle at $\mathrm{De}=1$ or 2 would have a low $\epsilon_{\text {eff }}^{\mathrm{EL}}$, i.e., it does not experience significant time in the strong region $\left(\mathrm{De}_{\text {local }}>1 / 2\right.$ ) of the extensional field. In contrast, a stretching DNA at the front of the obstacle can move very close to the obstacle surface (where the strain rate is maximum) and it can adopt long-lived (high strain) metastable configurations. The combined effects of the DNA configuration, DNA finite size, and the inhomogeneous strain rate explain the large difference we see in the extension of DNA leaving the obstacle at $\mathrm{De}=2$ and 9 . Importantly, this postimpact stretch is still limited by the post-impact strain, which is only in the range of $\epsilon^{\mathrm{EL}} \sim 1-1.5$. So, for larger De, we postulate that extensions occurring at the rear of the obstacle will not greatly exceed $40 \%$ (affine limit).

Finally, note that after the post-impact stretch, the molecules thermally relax back to an equilibrium coil as they move away from the obstacle. We show this explicitly in Figure 8c, by replotting the data to match the form of eq 10 . Here we subtract the mean square equilibrium stretch $\left(\left\langle x_{\mathrm{ex}}{ }^{2}\right\rangle_{0} / L^{2}=0.018\right)$ in order to see the exponential relaxation scaling for such small perturbations from equilibrium. The ensemble average longest relaxation time is equivalent to $\tau$, so that the average time it takes these molecules return to coil configurations is independent of $\mu \mathbf{E}$, as predicted.

\section{Conclusion}

This study shows how one can harness electric field gradients to manipulate flexible charged macromolecules. Specifically, we examined the deformation history of single DNA molecules in the electric field gradients near a large insulating post. On average, the molecules slightly stretch along the axis of extension before impact, but still showed characteristic configuration-sensitive extension-strain trajectories of an elongational field. Moreover, these stretched DNA affinely compress at the backside of the obstacle, and can restretch in the field direction as they leave the obstacle. This post-impact stretch was much more pronounced at $\mathrm{De}=9$ than $\mathrm{De}=2$, which we argued was due to the DNA's crumpled configuration, its finite size, and the steeply decaying inhomogeneous strain rate. Finally, the molecules thermally relax in low field gradient regions away from the obstacle. The terminal relaxation time is identically $\tau$.

More generally, we presented situations where the electrohydrodynamic equivalence is valid in free solution, as both electric field gradients and elongational flows create strong extensional fields. A more dramatic example of this equivalence would be to demonstrate coil-stretch hysteresis ${ }^{46}$ with electric field gradients. This study is also a unique analysis of deformation dynamics in an inhomogeneous (though pseudo-homogeneous) extensional field. We stress that electric field gradients provide a convenient way to induce purely extensional fields without shear components near surfaces.

Knowing how DNA molecules will extend and compress in field gradients offers a powerful new tool for microfluidic process design. One may consider the cylinder as a first order example; more complex obstacle geometries or channel contractions/expansions can be envisioned for custom DNA manipulations. Potential applications include pre-straining configurations, compression, or stretching of DNA. For example, we have observed history-dependent DNA deformation dynamics from "pre-crumpled" molecules. Additionally, we are able to quickly compress a stretched DNA molecule by introducing it to a stagnation point near a large obstacle. This may be a valuable tool for collision-based DNA mapping applications that rely on frequent transitions from stretched to compact configurations. But we also foresee creation of custom geometries to fully stretch DNA with electric fields so that nanoanalytical methods may be incorporated to decode or map gene locations. ${ }^{2}$ Preliminary experiments in contractions analogous to those used in Chan et al. ${ }^{2}$ have shown complete stretching of DNA.

Acknowledgment. This work was supported by NSF CAREER Grant No. CTS-0239012.

\section{References and Notes}

(1) Meller, A.; Nivon, L.; Branton, D. Phys. Rev. Lett. 2001, 86, 3435-3438.

(2) Chan, E. Y.; et al. Genome Res. 2004, 14, 1137-1146.

(3) Smith, S. B.; Cui, Y.; Bustamante, C. Science 1996, 271, 795799.

(4) Perkins, T. T.; Smith, D. E.; Larson, R. G.; Chu, S. Science 1995, 268, 83-87.

(5) Ferree, S.; Blanch, H. W. Biophys. J. 2003, 85, 2539-2546.

(6) Perkins, T. T.; Smith, D. E.; Chu, S. Science 1997, 276, 20162021.

(7) Smith, D. E.; Chu, S. Science 1998, 281, 1335-1340.

(8) Smith, D. E.; Babcock, H. P.; Chu, S. Science 1999, 283, 1724-1727.

(9) Babcock, H. P.; Teixeira, R. E.; Hur, J. S.; Shaqfeh, E. S. G.; $\mathrm{Chu}, \mathrm{S}$. Macromolecules 2003, 36, 4544-4548.

(10) Hur, J. S.; Shaqfeh, E. S. G.; Babcock, H. P.; Chu, S. Phys. Rev. E 2002, 66, 011915.

(11) Han, J.; Craighead, H. G. Science 2000, 288, 1026-1029.

(12) Duong, T. T.; et al. Microelectron. Eng. 2000, 67-68, 905912.

(13) Doyle, P. S.; Bibette, J.; Bancaud, A.; Viovy, J.-L. Science 2002, 295, 2237.

(14) Huang, L. R.; Silberzan, P.; Tegenfeldt, J. O.; Cox, E. C.; Sturm, J. C.; Austin, R. H.; Craighead, H. Phys. Rev. Lett. 2002, 89, 178301.

(15) Baba, M.; Sano, T.; Iguchi, N.; Iida, K.; Sakamoto, T.; Kawaura, H. App. Phys. Lett. 2003, 83, 1468-1470.

(16) Inatomi, K.; Izuo, S.; Lee, S.; Ohji, H.; Shiono, S. Microelectron. Eng. 2003, 70, 13-18.

(17) Randall, G. C.; Doyle, P. S. Phys. Rev. Lett. 2004, 93, 058102.

(18) Randall, G. C.; Doyle, P. S. Mater. Res. Soc. Symp. Proc. 2004, 790, P3.3.

(19) Tessier, F.; Labrie, J.; Slater, G. W. Macromolecules 2002, $35,4791-4800$.

(20) Olivera, B. M.; Baine, P.; Davidson, N. Biopolymers 1964, 2, 245-257.

(21) Bird, R. B.; Armstrong, R. C.; Hassager, O. Dynamics of Polymeric Liquids; Wiley: New York, 1987; Vol. 1.

(22) Deen, W. M. Analysis of Transport Phenomena; Oxford University Press: New York, 1998.

(23) Larson, R. G.; Magda, J. J. Macromolecules 1989, 22, 30043010 .

(24) Larson, R. G. J. Non-Newt. Fluid Mech. 2000, 94, 37-45.

(25) Long, D.; Dobrynin, A. V.; Rubinstein, M.; Ajdari, A. J. Chem. Phys. 1998, 108, 1234-1244.

(26) Barrat, J.-L.; Joanny, J.-F. Adv. Chem. Phys. 1996, 94, 1-66.

(27) Doi, M.; Edwards, S. F. The Theory of Polymer Dynamics; Clarendon Press: Oxford, U.K., 1988.

(28) Stigter, D.; Bustamante, C. Biophys. J. 1998, 75, 1197-1210.

(29) Nollert, M. U.; Olbricht, W. L. Rheol. Acta 1985, 24, 3-14.

(30) Szeri, A. J.; Wiggins, S.; Leal, L. G. J. Fluid Mech. 1991, 228, 207-241.

(31) Feng, J.; Leal, L. G. J. Non-Newt. Fluid Mech. 1997, 72, 187218. 
(32) Carrington, S. P.; Tatham, J. P.; Odell, J. A. Polymer 1997, 38, 4595-4607

(33) Panwar, A. S.; Kumar, S. J. Chem. Phys. 2003, 118, 925936.

(34) Xia, Y.; Whitesides, G. M. Angew. Chem., Int. Ed. Engl. 1998, $37,550-575$.

(35) Randall, G. C.; Doyle, P. S. Unpublished.

(36) Crocker, J. C.; Grier, D. G. J. Colloid Interface Sci. 1996, 179 298-310.

(37) Viovy, J.-L. Rev. Mod. Phys. 2000, 72, 813-872.

(38) Gutman, M.; Nachliel, E.; Kiryati, S. Biophys. J. 1992, 63, 274-280.

(39) Maier, B.; Rädler, J. O. Macromolecules 2000, 33, 7185-7194.

(40) Marko, J. F.; Siggia, E. D. Macromolecules 1995, 28, 87598770 .
(41) Bakajin, O. B.; Duke, T. A. J.; Chou, C. F.; Chan, S. S.; Austin, R. H.; Cox, E. C. Phys. Rev. Lett. 1998, 80, 2737-2740.

(42) Petersen, M.; Jacobsen, J. P. Bioconjugate Chem. 1998, 9, 331-340.

(43) Bensimon, A.; Simon, A.; Chiffaudel, A.; Croquette, V.; Heslot, F.; Bensimon, D. Science 1994, 265, 2096-2098.

(44) Jendrejack, R. M.; Dimalanta, E. T.; Schwartz, D. C. Graham, M. D.; de Pablo, J. J. Phys. Rev. Lett. 2003, 91, 038102.

(45) Inoué, S.; Spring, K. R. Video Microscopy; Plenum Press: New York, 1997.

(46) Schroeder, C. M.; Babcock, H. P.; Shaqfeh, E. S. G.; Chu, S Science 2003, 301, 1515-1519.

MA048073G 